\title{
A12 RHEUMATOID ARTHRITIS: INVOLVEMENT OF THE SYNOVIUM IN THE PRECLINICAL PHASE?
}

M J de Hair, ${ }^{1}$ M G van de Sande, ${ }^{1}$ W Bos, ${ }^{2}$ C van der Leij, ${ }^{3}$ M D Smith, ${ }^{4}$ M Maas, ${ }^{3}$ D van Schaardenburg, ${ }^{2}$ B A Dijkmans, ${ }^{5}$ D M Gerlag, ${ }^{1}$ P P Tak' ${ }^{1}$ Division of Clinical Immunology and Rheumatology, Academic Medical Center, University of Amsterdam, Amsterdam, Netherlands; ${ }^{2}$ Reade, Amsterdam, Netherlands; ${ }^{3}$ Department of Radiology, Academic Medical Center, University of Amsterdam, Amsterdam, Netherlands; ${ }^{4}$ Rheumatology Research Unit, Repatriation General Hospital, Adelaide, Australia; ${ }^{5}$ Department of Rheumatology, Free University Medical Centre, Amsterdam, Netherlands

\subsection{6/ard.2010.149096.12}

Background and objectives The aetiology of rheumatoid arthritis (RA), a prototype immune-mediated inflammatory disease, is poorly understood. Increased serum levels of acute phase reactants and autoantibodies (IgM rheumatoid factor (IgM-RF) and anticitrullinated protein antibodies (ACPA)) may be detected several years before the development of clinical signs and symptoms. Moreover, animal models of RA as well as examination of clinically uninvolved joints from established RA patients have suggested that clinically manifest arthritis may be preceded by an asymptomatic phase characterised by synovial inflammation. However, it is unknown whether the disease process starts in the synovium or at other sites in the body.

Materials and methods Fifty five individuals without any evidence of arthritis upon physical examination who were positive for IgM-RF and/or ACPA were included in the study and prospectively followed to assess arthritis development. To evaluate synovial inflammation, all participants underwent dynamic contrast enhanced MRI and arthroscopic synovial biopsy sampling of a knee joint at inclusion. An interim analysis of the first 13 individuals has been performed; these data are shown in this abstract. Results were compared with knee MRI data and synovial biopsy data of 6 and 10 healthy controls, respectively.

Results MRI findings evaluated by measurement of maximal enhancement, rate of enhancement, synovial volume and enhancement shape curve distribution were similar in the autoantibody positive individuals compared to the healthy controls. Consistent with these findings, all but one autoantibody positive individuals showed very low scores for $\mathrm{T}$ cells, B cells, intimal macrophages, sublining macrophages, fibroblastlike synoviocytes, plasma cells, E-selectin, ICAM-1, VCAM-1, and von Willlebrand factor, all in the same range as those in normal controls. The patient with higher scores showed patellofemoral joint space narrowing on conventional x-ray consistent with osteoarthritis.

During follow-up of a median of 37 (range 25-45) months, four individuals developed clinical signs and symptoms of arthritis. In these patients the baseline synovium was completely comparable with the synovium of healthy controls and of the individuals who did not develop arthritis. Data will be presented on the larger cohort. 
Conclusions Subclinical inflammation of the synovium does not coincide with the appearance of serum autoantibodies during the preclinical arthritis stage. Thus, systemic autoimmunity precedes the development of synovitis, suggesting that a 'second hit' is involved. Moreover, even if there are no signs of synovial inflammation, autoantibody positive individuals may suffer from arthralgia. 